\title{
An Overview of Functional Components of Artificial Intelligence Financial Decision Support System
}

\author{
Qi Wang \\ Accounting School, Nanfang College of Sun Yat-sen University, Guangzhou, China \\ Email: wangqijnu@163.com
}

How to cite this paper: Wang, Q. (2018) An Overview of Functional Components of Artificial Intelligence Financial Decision Support System. Open Journal of Social Sciences, 6, 115-126. https://doi.org/10.4236/jss.2018.68009

Received: July 31, 2018

Accepted: August 18, 2018

Published: August 21, 2018

Copyright $\odot 2018$ by author and Scientific Research Publishing Inc. This work is licensed under the Creative Commons Attribution International License (CC BY 4.0).

http://creativecommons.org/licenses/by/4.0/

\begin{abstract}
The development of artificial intelligence technology makes the intelligent decision-making of enterprise finance possible. Combined with the traditional enterprise financial decision support system, this paper summarizes and introduces the structure and function of the enterprise Financial Decision Support System and its subsystems under the development of artificial intelligence in detail.
\end{abstract}

\section{Keywords}

Artificial Intelligence, Financial Decision Support System, Structure and Function

\section{Introduction}

With the development of society, the company runs in a rapidly changing and unstable economy, and the enterprise faces the difficult of recording large amounts of management and operation data. The intensification of competition and the development of e-commerce require enterprises to have more accurate, timely and critical information, more in-depth financial analysis, more effective and timely communication and higher quality decision-making. Therefore, an enterprise financial decision support System (FDSS) was born at right moment. With the development of artificial intelligence technology, the FDSS combined with artificial intelligence technology becomes a research hotspot [1] [2]. In 2005, Jiangsu Land Reclamation Group developed an advanced FDSS, which integrated the reporting standardization, intelligent decision and information network. The FDSS provided convenient and effective financial information support for making decision of enterprise. Artificial intelligence financial deci- 
sion support system (AIFDSS) has been attracted many attention and applied for large enterprise groups.

Decision Support System (DSS) is a computer application system that can assist and support managers to make decisions and improve the effectiveness of decisions [3]. FDSS is a computer system formed by applying DSS design ideas and methods to financial management. FDSS is a computer system used by financial personnel to assist decision-making in financial management, financial control, financial analysis, financial forecasting and decision-making. The main decision-making problems in FDSS include [1]:

1) Structural decision making. Routine repetitive decision-making, with certain rules to follow, can make an orderly arrangement in advance to achieve the desired results and goals. It is usually possible to describe and solve problems by conventional quantitative mathematical methods.

2) Unstructured decision-making. It has never happened before, or the decision-making process structure is too complex to follow the rules, or it is especially difficult to describe and solve the decision-making problems that must be solved immediately once the key problems appear.

3) Semi-structured decision-making is a decision-making problem between structured decision-making and unstructured decision-making, which has two decision-making characteristics.

The traditional financial decision support system mainly consists of two parts: database and model library [3]. There is often little that can be done about semi-structured or unstructured problems.

Whereas, AIFDSS has a breakthrough in solving semi-structured or unstructured problems. The main difference between FDSS of artificial intelligence and the traditional FDSS structures is that the former joins knowledge-based systems and other business intelligence technologies to simulate human's peculiar thinking ability. It can not only solve the problem of conventional quantitative nature, but also can help decision-makers to recognize the decision environment, clear targets and solve the semi-structured or unstructured problems by providing background materials, helping clear the problem, modifying and perfecting the model, enumerating the possible schemes and analyzing and comparing [4]. Its features are as follows: 1) it has inference structure and can simulate the thinking process of decision makers; 2) it has learning function and increases the knowledge automatically. Therefore, it can be improved slowly with little or without intermediate maintenance; 3) solve semi-structured unstructured problems of financial decision-making more effectively; 4) it can track the solution process of the problem, prove the correctness of the financial model scheme, and enhance the credibility of the financial decision-making model scheme [5].

Due to the wide application of AIDFSS, a lot of researches on its functional components and new technologies are emerging. In 1980, Sprague proposed a three-component theory, which includes dialogue component, data component and model component. It was the based theory of DSS and played a significant 
role in the subsequent development of DSS. In 1981, Bonczek proposed a three systems structure, namely the language system (LS), the problem processing system (PPS) and the knowledge system (KS). This structure has its characteristic in dealing with problem, which makes it have a better effect, and henceforth lay the foundation for intelligent decision-making system development. In the late 1980s, with the rise of artificial neural network and machine learning technology, integrated the artificial intelligence into the DSS to form traditional intelligent decision support system. With the application of expert systems technology, DSS can be used better in human's knowledge, such as problem description knowledge, process knowledge and deductive knowledge for solving problem [6]. In addition, the development of artificial intelligence technology, expert system technology, database technology and internet/intranet technology provides powerful technical support for intelligent decision support system. Thus, some representative decision support technical tools were produced, including data warehouses [7] [8] [9], on-line analytical processing, data mining [10], ANN [11], genetic algorithm and fuzzy logic [12], which had a great influence on the evolution and development of the structure of intelligent decision support system.

The above literatures mainly focus on the design and function of one part. Few research studies all the components of the structure systematically. Therefore, in order to make up the literature gap, this paper studies the influence of each component and its sub-components on decision-making in the practical application of enterprises.

\section{Artificial Intelligence Financial Decision Support System (AIFDSS)}

From the perspective of the logical structure, intelligent decision support system generally can be divided into three parts: database, model base and knowledge base [13]. In the 1990s, the concept of data Warehouse, which combined with the traditional Intelligent FDSS further improved the effect of the auxiliary decision-making [8].

Intelligent decision support systems are integrated by model bases, data warehouses, OLAP technologies, data mining, expert system and interactive interfaces. The data warehouse can realize the storage and synthesis of decision topics. OLAP technologies implement multidimensional data analysis, data mining is used to mine knowledge in database and data warehouse, model base realizes the combined aid decision of multiple generalized models, and expert system uses knowledge reasoning to achieve qualitative analysis. They complement each other, rely on each other, give full play to their respective auxiliary decision-making advantages and realize more effective auxiliary decision-making.

As the data warehouse has become an indispensable part of intelligent decision support system, it has been the prerequisite for it to make higher quality 
decisions [8] [14], and this paper mainly studies the requirements of AIFDSS on its components in the operation process. therefore, get rid of the business intelligence technology (OLAP and data mining), basing on the Efraim \& Jay [5] proposed the basic structure of the decision support system, added data warehouse module, AIFDSS can be divided into data management subsystem, data Warehouse and model management subsystem and knowledge management subsystem of four parts, as shown in Figure 1.

Each component will be discussed in detail in the following sections.

\section{Data Management Subsystem}

Data management subsystem is the basis of decision analysis of AIFDSS.A system that stores relevant data for a particular situation consists of the following elements: database, database management system, data catalog and query function.

1) Database

The database collects relevant data to meet the needs and structures of the organization and can be used by more users for more purposes. Data can be raw or generalized, including documents, images, maps, sound, video, and animations, concepts and ideas. Data sources can be divided into internal data sources, external data sources and personal data sources. The storage structure of database can be roughly divided into three categories: relational database, hierarchical database and network database [15]. In fact, the backstage of most management information systems is carried out in relational databases, such as Oracle and MS.

2) Data base management system

DBMS creates, users, and updates databases. An effective database and its management can support many management activities. Typical examples are browsing records, creating and maintaining a diverse set of data relationships,

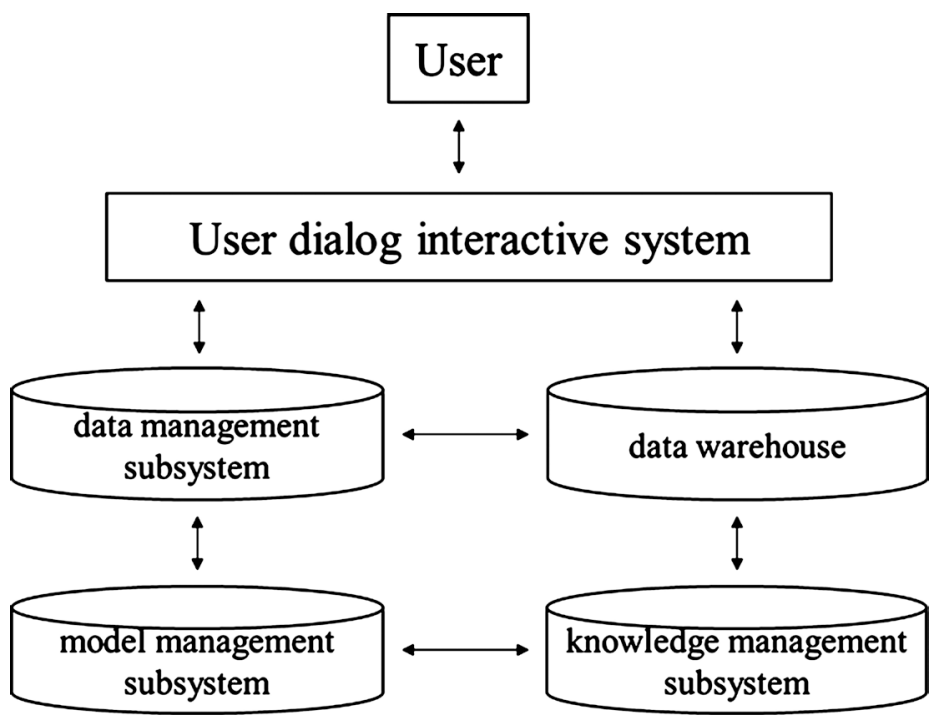

Figure 1. Schematic diagram of main structure of AIFDSS. 
and generating reports. The specific functions of the database management system are shown in Table 1.

3) Data catalog

A data catalog is a directory of all the data in a database. And it is a place where the database server stores data files, including not only the files on the table, but also the data files and server options files. The main function is to answer questions about data availability and the exact meaning of data source. Liking other directories, this directory supports adding new items, deleting items, and restoring information about specific objects.

4) Query function

The main tasks of the query function are to use, process, and query data. It accepts data requests from other DSS components, determines how those requests are met, explicitly expresses detailed requests and returns the results to the requester.

\section{Data Warehouse}

In essence, data warehouse technology is an information integration technology. The data warehouse obtains the original data from multiple information sources. These raw data are stored in the internal database of the data warehouse after sorting and processing, providing users with a unified, coordinated and integrated information environment, supporting the overall decision-making process of the enterprise and in-depth and comprehensive analysis of enterprise operation and management [7] [8] [9]. To achieve this goal, a data warehouse generally consists of the following seven main components [16]:

1) Source data

Source data is provided for the data warehouse, such as operational data of various production system databases, online transaction processing system (OLTP) and external data sources, which can all be used as data sources of the data warehouse.

2) Extraction, transformation and load tools

Its function is to extract data from the data source, check and organize the data, reorganize and process the data according to the design requirements of the data warehouse, and load it into the target database of the data warehouse.

3) Data modeling tool

The modeling tool establishes an information model for the source database of the data warehouse and the target database.

4) Central repository and data mart

This part is used to store data models and metadata. Meta data describes the information of the source data and the target data itself in the data warehouse, defines the transformation process from the source data to the target data, and is used to store the reorganized and sorted data. In some very large scale decision support applications, dedicated multidimensional databases have certain advantages. A data mart is a collection of DSS applications that supports a portion or 
specific business requirement. The data in the data mart still has the characteristics of data warehouse, except that the data in the data mart is customized for a certain department or a specific business need [17].

5) Target database

The target database is used to store tested, sorted, processed, and reorganized data.

6) Front-end data access and analysis tools

Front-end data access and analysis tools provide business analysts and decision makers with access to data in the target database for further in-depth analysis.

7) Data warehouse management tools

Data warehouse management tools provide management means for data warehouse operation, including security management, storage management and other aspects.

We can see that in a data warehouse, the source data comes from the existing production system. As long as the data warehouse can provide the necessary information for the decision-making and analysis process supported by the data warehouse, it may become the data source of the data warehouse. Therefore, the data warehouse needs tools to extract data from the data source. Data must be validated before it can enter the data warehouse to eliminate errors that may be hidden in the data. In order to meet the need of decision support and in-depth analysis, data should pass special processing and reorganize, after inspection, sorting, processing and reorganizing, the data will be loaded into one or more of the data warehouse database, all these works are made by data extraction and conversion tools. In order to describe the requirements for data validation, collation, processing as well as the corresponding processes and steps, there must be data modeling tools.

\section{Model Management Subsystem}

The model management subsystem consists of the following elements: model base, model management system, modeling language, model directory, model execution, composition and instruction processor [18] [19].

1) Model base

The model library includes general and specialized statistical, financial, forecasting, management science, and other types of quantitative models. These models provide analytical capability for DSS. The model base has ability to invoke, run, modify, compose, and examine models. The models in the model base are divided into four types: strategic, tactical, operational, and analytical. In addition, there are model infrastructure artifacts and programs.

Model building module is a tool for data analysis, including the basic construction and program of the model, such as random number production program, curve or line fitting program, net present value rapid program and regression analysis. It can be applied not only directly as a model, but also as part of a 
larger model.

2) Modeling tool

When encountering semi-structured or unstructured problems, some model tools are needed, such as programming tools and language programming models, such as C++, Java, OLAP software, or spreadsheet (Excel).

3) Model base management system

The function of model base management system software is to use programming language, DSS tools or subroutines and other basic components to create models, generate new programs and reports, replace and transform models, and control model data. The specific functions are shown in Table 1.

4) Model catalog

The role of the model catalog is similar to that of the data catalog, which is the classification of all models. It includes model definition. The main function is to answer questions about the feasibility and function of the model.

5) Execution, composition and instructions

Model execution controls the actual operation of the model. Model composition includes the operation of integrating some models as needed (for example, managing the output of a model; For example, predictions are processed by another model, such as the linear programming model). The model instruction is used to accept and interpret instructions from the user interface portion and transmit them to the MBMS, model execution, or integration functions. Because in many cases, DSS systems do not know which models are used for which situations, these operations are typically assisted by knowledge system components and humans in intelligent financial DSS [20].

\section{Knowledge Management Subsystem}

The knowledge management subsystem provides professional knowledge to solve many unstructured and even semi-structured problems, which is the biggest difference between the traditional FDSS and the AIFDSS. The knowledge management subsystem can also solve problems that cannot be solved by other decision support system components before and improve their operational execution ability. Its core system is the expert system [21] [22].

The name expert system derives from the term knowledge-based expert system. Expert systems use human knowledge acquired in computers to solve problems that usually require human expertise [6]. In every expert system, there are three main components: knowledge base, inference engine and user interface. The expert system that communicates with users may also include the following additional components: the knowledge acquisition subsystem, blackboard structure, explain subsystem, knowledge-refining system.

1) Knowledge acquisition

Knowledge acquisition is the accumulation, transfer and transformation of expertise from experts or records of knowledge sources to computer programs used to build or expand knowledge bases. Potential sources of knowledge include 
Table 1. Components and Technology of AIFDSS.

\begin{tabular}{|c|c|c|}
\hline Financial DSS components & Sub-components & Definitions and Functions \\
\hline \multirow{4}{*}{$\begin{array}{l}\text { Database management } \\
\text { subsystem [5] [15] }\end{array}$} & database & Collect and store relevant data. \\
\hline & DBMS & $\begin{array}{l}\text { Get and extract data from the database, update (add, delete, edit, modify) data records and } \\
\text { documents. Provide comprehensive data security for queries and reports, handle personal } \\
\text { and informal data, perform complex data processing tasks on a query basis, record data } \\
\text { usage, and manage data. }\end{array}$ \\
\hline & data catalog & $\begin{array}{l}\text { Data accessibility of the answer; Answer data sources; The exact meaning of the answer } \\
\text { data; Add, delete items, and fix feature objects. }\end{array}$ \\
\hline & query facility & Query data; Processing data; Display data results. \\
\hline \multirow[t]{6}{*}{$\begin{array}{l}\text { data warehouse [7] [8] [9] } \\
{[10][16][23]}\end{array}$} & source data & $\begin{array}{l}\text { Source data is provided for the data warehouse, such as various production system } \\
\text { databases, operational data of online transaction processing system (OLTP), external data } \\
\text { sources, etc. }\end{array}$ \\
\hline & $\begin{array}{l}\text { Extraction, } \\
\text { transformation and load } \\
\text { tools }\end{array}$ & $\begin{array}{l}\text { Data is extracted from the data source, tested and sorted out. According to the design } \\
\text { requirements of the data warehouse, data is reorganized and processed and loaded into the } \\
\text { target database of the data warehouse. }\end{array}$ \\
\hline & data modeling tool & Model information for the source database of the data warehouse and the target database. \\
\hline & $\begin{array}{l}\text { central repository and } \\
\text { data mart }\end{array}$ & Store data models and metadata. Includes metadata, data models, and data marts. \\
\hline & target database & Store tested, sorted, processed, and reorganized data. \\
\hline & $\begin{array}{l}\text { Data warehouse } \\
\text { management tools }\end{array}$ & $\begin{array}{l}\text { Provide management means for data warehouse operation, including security } \\
\text { management, storage management and other aspects. }\end{array}$ \\
\hline \multirow{6}{*}{$\begin{array}{l}\text { Model base management } \\
\text { subsystem [24] [25] [26] }\end{array}$} & model base & Storage strategy, tactics, operations, analysis models. \\
\hline & model building blocks & $\begin{array}{l}\text { Data analysis tools, including model infrastructure and programs, can be applied directly } \\
\text { as models or as components of large models. }\end{array}$ \\
\hline & modeling tool & $\begin{array}{l}\text { Deals with semi-structured or unstructured issues such as programming tools and } \\
\text { language programming models. Typical examples are spreadsheets (Excel). }\end{array}$ \\
\hline & MBMS & $\begin{array}{l}\text { Easily and quickly create the model; Storing, acquiring, and managing multiple different } \\
\text { types of models in a logical and integrated manner; Use and integrate infrastructure } \\
\text { components; Categorize and display model catalogs; Record model data and application } \\
\text { usage; Link integration database and model library; Manage and maintain model libraries: } \\
\text { store, use, run, update, connect, categorize, and query; Use multiple models to support } \\
\text { problem solving. }\end{array}$ \\
\hline & model catalog & Model classification; Model definition; Answer model feasibility and function. \\
\hline & $\begin{array}{l}\text { Execution, composition } \\
\text { and instructions }\end{array}$ & $\begin{array}{l}\text { Control the actual running process of the model; Accept and interpret instructions from } \\
\text { the user interface section and send them to the MBMS, model execution. }\end{array}$ \\
\hline \multirow{7}{*}{$\begin{array}{l}\text { Knowledge management } \\
\text { subsystem (expert system) } \\
{[6][21][27]}\end{array}$} & knowledge acquisition & $\begin{array}{l}\text { Explain and integrate answers to questions through knowledge engineering. Create clear } \\
\text { concept of analogy and construction counterexample. }\end{array}$ \\
\hline & knowledge base & Understand and expound knowledge; Knowledge required to solve problems. \\
\hline & inference engine & $\begin{array}{l}\text { Reasoning information in knowledge base and blackboard structure, controlling structure } \\
\text { and rule interpreter. }\end{array}$ \\
\hline & user interface & Provide communication between users and computers. \\
\hline & blackboard & Describe specific problems; Document assumptions and decisions. \\
\hline & explanation subsystem & Source of follow-up conclusions; Explain expert system behavior. \\
\hline & $\begin{array}{l}\text { knowledge-refining } \\
\text { system }\end{array}$ & $\begin{array}{l}\text { Analyze the knowledge and application, generate more accurate knowledge base and } \\
\text { effective reasoning method. }\end{array}$ \\
\hline
\end{tabular}


human experts, textbooks, multimedia files, databases (public or private), special research reports, and information available from the web. Acquiring knowledge through experts is a very complex task. In the process of building a large system, we need a knowledge engineering or knowledge enlightening expert to communicate with one or more human experts. Typically, knowledge engineering helps experts build problem areas by interpreting and integrating the answers to human problems, proposing analogies, and constructing counterexamples to counter conceptual clarity.

2) Knowledge base

The knowledge base contains the knowledge needed to understand, explain, and solve problems. It includes two essential elements: a) Facts, such as problems and theories in the problem domain; b) Special heuristic algorithms or rules guiding knowledge to be applied in special fields to solve specific problems.

3) Inference engine

The "brain" of an expert system is an inference engine, also known as a control structure or rule interpreter. The component is essentially a computer program that provides a way to reason about information in the knowledge base and blackboard structures and draw conclusions.

4) User interface

The expert system provides a language processor for friendly problem communication between users and computers. This communication can be carried out through natural language. Due to the limitations of technology, most current systems use questions-and-answers to communicate with users. Sometimes it is supplemented by menus, spreadsheets, and graphics.

5) Blackboard

Blackboard architecture belongs to the field of working memory. It is used as a database to make a specific description of the current problem by entering data. It is also used to record the assumptions and decisions in the middle. Three types of decisions can be recorded in the blackboard structure: planning (how to solve a problem), agenda (potential action to be executed), and solution (candidate assumptions and action processes generated by the system so far).

6) Explanation subsystem

The ability to track the sources of conclusions is critical in the process of experience transfer and problem solving. The interpretation subsystem can track this responsibility and explain the behavior of the expert system through interactive questioning, asking the following questions: why does the expert system ask a question? How does a conclusion come about? Why would you reject other options? What is the plan to get the solution?

7) Knowledge-refining system

Human experts have a system of knowledge-refining system, that is, they can analyze their knowledge and its application, learn from it and improve gradually. Likewise, such assessments are necessary in computerized learning so that programs can analyze the reasons for their success or failure. This leads to a more 
accurate knowledge base and more efficient reasoning.

In conclusion, the functional structure of Financial Intelligence DSS can be listed in Table 1.

\section{Conclusion}

Due to the rapid development of artificial intelligence technology and the trend of promoting artificial intelligence in China in recent years, this paper mainly summarizes the functional structure and sub-components of enterprise financial decision support system under artificial intelligence. The main difference between AIFDSS and traditional FDSS is that the knowledge management subsystem (expert system) component is added to enable it to have some human reasoning and learning ability, so as to solve some structural or semi-structural problems. Therefore, when introducing the AIFDSS structure, this paper introduces the expert system in detail on the basis of introducing the basic construction and elaborates the relationship between them. The addition of expert system makes a qualitative leap in the auxiliary role of enterprise FDSS to the management decision maker, which can make the decision maker to solve the problem more clearly and quickly. In China, AIFDSS is still in the theoretical conceptual stage, and many technologies have not been applied and popularized. The research in this paper can provide some theoretical basis for researchers and pave the way for future practical research.

\section{Conflicts of Interest}

The authors declare no conflicts of interest regarding the publication of this paper.

\section{References}

[1] Feng, J. (1998) Research on Enterprise Financial Management Decision Support System. China Education Informatization, 8-10+17.

[2] Pan, H.P. (2016) Application Research of Intelligent Financial Decision Support System. Fujian Computer, 32, 100-101.

[3] Gorry, G.A. and Morton, M.S.S. (1970) Management Decision Systems: A Framwork for Management Information Systems. International Journal of Energy Research, 35, 781-794.

[4] Xie, K. (2003) Research on Financial and Accounting Intelligence Systems. Financial and Accounting Journal, 2b, 59-60.

[5] Turban, E., Aronson, J.E. and Liang, T.-P. (2009) Decision Support Systems and Intelligent Systems. Machinery Industry Press, Beijing.

[6] Liao, S.H. (2005) Expert System Methodologies and Applications-A Decade Review from 1995 to 2004. Expert Systems with Applications, 28, 93-103. https://doi.org/10.1016/j.eswa.2004.08.003

[7] Agosta, L. (2000) The Essential Guide to Data Warehousing. Prentice Hall PTR, Upper Saddle River.

[8] Inmon, W.H. (2002) Building the Data Warehouse. 3rd Edition, John Wiley \& Sons, Inc., New York City. 
[9] O'Brien, J. and Marakas, G. (2009) Management Information Systems. Systems Approaches for Sustainable Agricultural Development, 5, 439-446.

[10] Albashrawi, M. (2016) Detecting Financial Fraud Using Data Mining Techniques: A Decade Review from 2004 to 2015. Journal of Data Science, 14, 553-570.

[11] Kraus, M. and Feuerriegel, S. (2017) Decision Support from Financial Disclosures with Deep Neural Networks and Transfer Learning. Decision Support Systems, 104, 38-48. https://doi.org/10.1016/j.dss.2017.10.001

[12] Korczak, J., Hernes, M. and Bac, M. (2015) Fuzzy Logic in the Multi-Agent Financial Decision Support System. Federated Conference on Computer Science and Information Systems, Łódź, 13-16 September 2015, 1367-1376.

[13] Turban, E. and Aronson, J. (2000) Decision Support Systems and Intelligent Systems . Tsinghua University Press, Beijing.

[14] Peng, M.G. (2002) Data Warehouse Technology and Implementation. Electronic Industry Press, Beijing.

[15] Castelli, V. and Bergman, L.D. (2002) Chapter 10, Introduction to Content-Based Image Retrieval-Overview of Key Techniques. John Wiley \& Sons, Inc., New York City.

[16] Tang, S.W. and Pei, J. (1998) Data Warehouse Technology Seminar (3) Data Warehouse Architecture. China Financial Computer, 10-14.

[17] Yang, G., Zhang, L. and Ai, B. (2000) Data Warehouse and On-Line Analytical Processing Technology. Computer Engineering and Science, 22, 39-42.

[18] Han, J. and Kamber, M. (2006) Data Mining: Concepts and Techniques. 2nd Edition, 5, 1-18.

[19] Han, J., Kamber, M. and Pei, J. (2011) Data Mining: Concepts and Techniques. Morgan Kaufmann, Burlington, 5, 394-395. (In Chinese)

[20] Respício, A., Captivo, M.E. and Rodrigues, A.J. (2002) A DSS for Production Planning and Scheduling in the Paper Industry. In: Adam, F., Brezillon, P., Humphreys, P. and Pomerol, J.-C., Eds., Decision Making and Decision Support in the Internet Age, Oak Tree Press, Cork, 298-308.

[21] Nedović, L. and Devedžić, V. (2002) Expert Systems in Finance-A Cross-Section of the Field. Expert Systems with Applications, 23, 49-66. https://doi.org/10.1016/S0957-4174(02)00027-1

[22] Nurminen, J.K., Karonen, O. and Hätönen, K. (2003) What Makes Expert Systems Survive over 10 Years-Empirical Evaluation of Several Engineering Applications. Expert Systems with Applications, 24, 199-211. https://doi.org/10.1016/S0957-4174(02)00149-5

[23] Nemati, H.R. and Barko, C.D. (2008) Organizational Data Mining (ODM): An Introduction. In: Nemati, H. and Barko, C.D., Eds., Organizational Data Mining. Leveraging Enterprise Data Resources for Optimal Performance, IGI Global, Hershey, $1-8$.

[24] Sprague, R.H. (1980) A Framework for the Development of Decision Support Systems; Society for Information Management and the Management Information Systems Research Center.

[25] Hand, D.J., Mannila, H. and Smyth, P. (2001) Principles of Data Mining (Adaptive Computation and Machine Learning). MIT Press, Cambridge.

[26] Mining, W.I.D. (2006) Data Mining: Concepts and Techniques. Morgan Kaufmann, Burlington. 
[27] Prasad, R., Ranjan, K.R. and Sinha, A.K. (2006) AMRAPALIKA: An Expert System for the Diagnosis of Pests, Diseases, and Disorders in Indian Mango. Knowledge-Based Systems, 19, 9-21. https://doi.org/10.1016/j.knosys.2005.08.001 